\title{
Two-Dimensional Exchange Deuteron NMR of Polystyrene and Poly(vinyl methylether) Blend
}

\author{
Kunio HIKICHI, Akinori TeZuKa, ${ }^{\dagger}$ and $\mathrm{K}$. TaKegoshi ${ }^{\dagger \dagger}$ \\ Division of Biological Sciences, Graduate School of Science, \\ Hokkaido University, Sapporo 060-0810, Japan
}

(Received November 20, 1997) KEY WORDS 2D Exchange Nuclear Magnetic Resonance / Deuteron / Polystyrene / Polyvinyl(methyl
ether) / Blend /

The physical properties of polystyrene/poly(vinyl methylether) (PS/PVME) blend have attracted much interest and been studied by many methods. In previous papers we reported the results of deuteron quadrupole echo NMR of main-chain deuterated polystyrene $\left(\mathrm{PS}-d_{3}\right)$ in the blend. ${ }^{1,2}$

Spiess et al. showed that two-dimensional (2D) exchange deuteron NMR spectroscopy is quite useful for detecting molecular motion in solid polymers. ${ }^{3}$ In this note we apply this technique to demonstrate molecular motion of polystyrene in blends.

PS- $d_{3}$ was synthesized by anionic polymerization of styrene- $d_{3}$, purchased from Cambridge Isotope Laboratories. The molecular weight determined by intrinsic viscosity is 260000 . PVME was obtained from Aldrich Chemical Company, Inc. The molecular weight determined by the intrinsic viscosity is 44000 .

The pure polystyrene and samples at a weight rario of $1: 1$ were cast from benzene solution of a concentration of $3 \mathrm{~g} / 100 \mathrm{ml}$ on a glass plate at room temperature. The resulting films were dried under vacuum at $322 \mathrm{~K}$ for more than 6 days.

Deuteron NMR measurement was made using a modified JEOL JNM-GX270 spectrometer operating at 41.7 MHz. A fast (up to $20 \mathrm{MHz}$ ) A/D converter (AP501 Autonics) and a pulse programmer developed at Physics Department, University of British Columbia, were installed. The 90 degree pulse length was $2.2 \mu \mathrm{s}$. We used a pulse sequence of $2 \mathrm{D}$ exchange NMR reported by Spiess et al. ${ }^{3}$ The mixing time of $2 \mathrm{D}$ exchange NMR was $10 \mathrm{~ms}$. The temperature of sample was controlled by gas flow from room temperature to $393 \mathrm{~K}$.

Figures 1 and 2 show the 2D exchange NMR spectra of pure PS at $353 \mathrm{~K}$ and at $393 \mathrm{~K}$, respectively. Onedimensional (1D) spectra are also depicted on the top of each spectrum. The 1D spectra show typical powder patterns which indicate that molecular motion is too slow to average out the quadrupole interaction.

The $2 \mathrm{D}$ spectrum at $353 \mathrm{~K}$ is localized on the diagonal without appreciable scatter. This means that the C-D bond of PS main chain does not undergo reorientational motion from one direction to another for a period of mixing time $(10 \mathrm{~ms})$, since the resonance position of deuteron is determined by the orientation of $\mathrm{C}-\mathrm{D}$ bond.

The spectrum at $393 \mathrm{~K}$ diffuses from the diagonal toward both axes and shows noticeable cross-peaks. The $1 \mathrm{D}$ spectrum is just the same as that at $353 \mathrm{~K}$. The results indicate that PS molecules in the blend undergo reorientational motion fast enough to change the

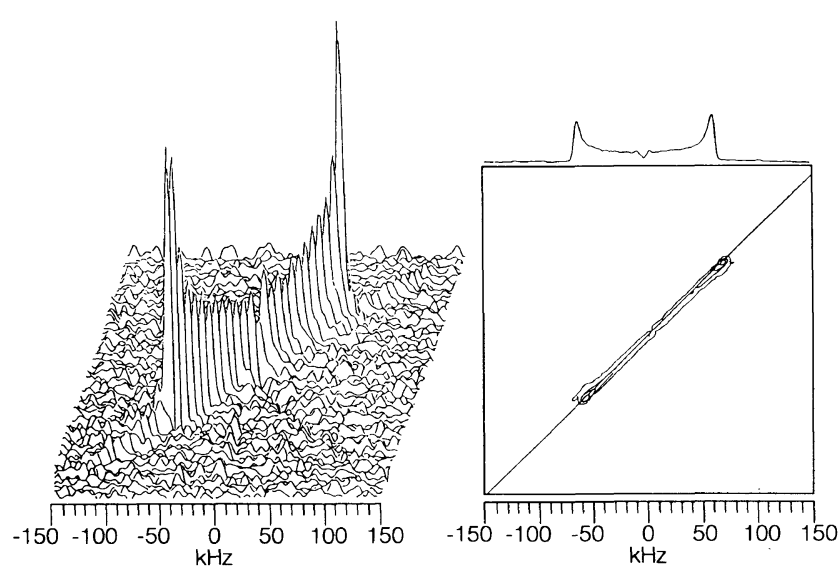

Figure 1. Stacked plots (left) and contour plots (right) of the twodimensional exchange deuteron NMR spectra of main-chain deuterated polystyrene at $353 \mathrm{~K}$.

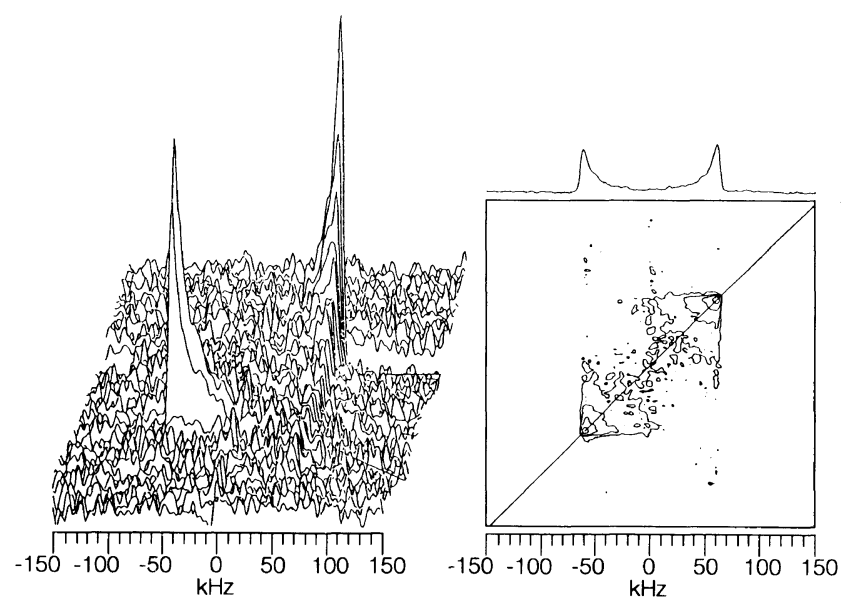

Figure 2. Stacked plots (left) and contour plots (right) of the two-dimensional exchange deuteron NMR spectra of main-chain deuterated polystyrene at $393 \mathrm{~K}$.

\footnotetext{
† Present address: Varian Japan, Ltd., Sumitomo Shibaura Bldg, Shibaura 4-16-36, Minato-ku, Tokyo 108-0023, Japan.

${ }^{+\dagger}$ Present address: Department of Chemistry, Graduate School of Science, Kyoto University, Kyoto 606-8224, Japan.
} 


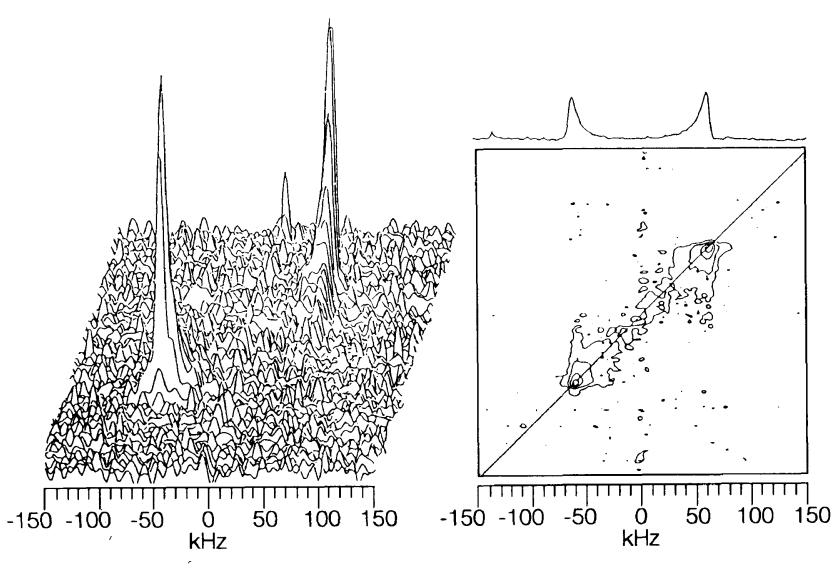

Figure 3. Stacked plots (left) and contour plots (right) of the two-dimensional exchange deuteron NMR spectra of a $1: 1$ blend of deuterated polystyrene and poly(vinyl methylether) at $393 \mathrm{~K}$. orientation in $10 \mathrm{~ms}$ but not so fast to average out the quadrupole interaction of an order of $100 \mathrm{kHz}$.

Figure 3 shows the spectrum of the blend at $333 \mathrm{~K}$. The 1D spectrum shows a powder pattern due to quadrupole interaction. The 2D spectrum shows exchange cross-peaks. It is clear that PS molecules in the blend undergo reorientation at frequencies higher than $100 \mathrm{~Hz}$ and lower than $100 \mathrm{kHz}$ at $333 \mathrm{~K}$ well below the glass transition temperature of pure PS.

It is well known that PS and PVME form compatible blend and mix together on a molecular scale. PS in the blend is embedded in a soft PVME matrix and is much more mobile than pure PS, as supported by present results.

\section{REFERENCES}

1. Akinori Tezuka, K. Takegoshi, and Kunio Hikichi, J. Mol. Struct., 355, 1 (1995).

2. Akinori Tezuka, K. Takegoshi, and Kunio Hikichi, J. Mol. Struct., 355, 9 (1995).

3. S. Wefing, S. Kaufmann, and H. W. Spiess, J. Chem. Phys., 89, 1234 (1988). 